\title{
Real-time phase-contrast flow MRI of haemodynamic changes in the ascending aorta and superior vena cava during Mueller manoeuvre
}

\author{
M. Fasshauer ${ }^{\text {a,d }}$, A.A. Joseph ${ }^{\text {b,d }}$, J.T. Kowallick ${ }^{\text {a,d }}$, \\ C. Unterberg-Buchwald ${ }^{\text {a,c,d }}$, K.D. Merboldt ${ }^{\mathrm{b}, \mathrm{d}}$, D. Voit ${ }^{\mathrm{b}, \mathrm{d}}$, \\ M. Steinmetz ${ }^{\text {d, e }}$, W. Staab ${ }^{\mathrm{a}, \mathrm{d}}$, S. Schaetz ${ }^{\text {b,d }}$, S. Zhang ${ }^{\text {b, }}$, J. Frahm ${ }^{\text {b, d, }}$, \\ J. Lotz ${ }^{\mathrm{a}, \mathrm{d}, *}$, J.M. Sohns ${ }^{\mathrm{a}, \mathrm{d}}$ \\ a Institute for Diagnostic and Interventional Radiology, Heart Centre, University Medical Centre, \\ Georg-August-University Göttingen, Germany \\ ${ }^{\mathrm{b}}$ Biomedizinische NMR Forschungs GmbH am Max-Planck-Institut für biophysikalische Chemie, Göttingen, Germany \\ ${ }^{\mathrm{C}}$ Clinic for Cardiology and Pneumology, Heart Centre, University Medical Centre, Georg-August-University Göttingen, \\ Germany \\ d DZHK, German Centre for Cardiovascular Research, Göttingen, Germany

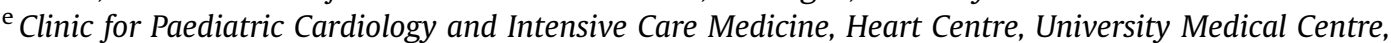 \\ Georg-August-University Göttingen, Germany
}

\section{ARTICLE INFORMATION}

\section{Article history:}

Received 30 April 2014

Received in revised form

1 June 2014

Accepted 3 June 2014
AIM: To evaluate the potential of real-time phase-contrast flow magnetic resonance imaging (MRI) at $40 \mathrm{~ms}$ resolution for the simultaneous determination of blood flow in the ascending aorta (AA) and superior vena cava (SVC) in response to reduced intrathoracic pressure (Mueller manoeuvre).

MATERIALS AND METHODS: Through-plane flow was assessed in 20 healthy young subjects using real-time phase-contrast MRI based on highly undersampled radial fast low-angle shot (FLASH) with image reconstruction by regularized non-linear inversion. Haemodynamic alterations (three repetitions per subject $=60$ events) were evaluated during normal breathing (10 s), inhalation with nearly closed epiglottis (10 s), and recovery (20 s).

RESULTS: Relative to normal breathing and despite interindividual differences, reduced intrathoracic pressure by at least $30 \mathrm{mmHg}$ significantly decreased the initial peak mean velocity (averaged across the lumen) in the AA by $-24 \pm 9 \%$ and increased the velocity in the SVC by $+28 \pm 25 \%$ ( $p<0.0001, n=23$ successful events). Respective changes in flow volume per heartbeat were $-25 \pm 9 \%$ in the AA and $+49 \pm 44 \%$ in the SVC $(p<0.0001, n=23)$. Flow parameters returned to baseline during sustained pressure reduction, while the heart rate was elevated by $10 \%(p<0.0001)$ after the start $(n=24)$ and end $(n=17)$ of the manoeuvre.

\footnotetext{
* Guarantor and correspondent: J. Lotz, Institute for Diagnostic and Interventional Radiology, University Medical Centre, Georg-AugustUniversity Göttingen, Robert-Koch-Str. 40, 37099 Göttingen, Germany. Tel.: +4955139 8965; fax: +49551399606.

E-mail address: Joachim.lotz@med.uni-goettingen.de (J. Lotz).
} 
CONCLUSIONS: Real-time flow MRI during low intrathoracic pressure non-invasively revealed quantitative haemodynamic adjustments in both the AA and SVC.

(c) 2014 The Royal College of Radiologists. Published by Elsevier Ltd. All rights reserved.

\section{Introduction}

Physiological manoeuvres that alter the intrathoracic pressure serve as non-invasive tests of cardiac function. In particular, during reduced intrathoracic pressure, i.e., the so-called Mueller manoeuvre (MM) with deep inspiration against the nearly closed epiglottis, echocardiography demonstrated an increased venous return and higher right ventricular stroke volume. ${ }^{1,2}$ A decrease of the intrathoracic pressure increases the pressure gradient between the thoracic cavity and the head and neck as well as the abdominal cavity. These alterations lead to an increase in systemic venous return and preload of the right ventricle. As the preload increases, the stroke volume of the right heart, via the Frank-Starling law, increases as well. Transient elevation of blood pressure in the pulmonary artery is a logical consequence. In addition, reduced intrathoracic pressure also decreases the initial pulmonary flow return to the left ventricle, which increases the pressure in the pulmonary artery even more, while momentarily decreasing the left-ventricular stroke volume.

To this regard, MM mimics some of the alterations observed during obstructive sleep apnoea (OSA). ${ }^{3,4}$ OSA is closely associated with diseases or symptoms such as sudden cardiac death, ${ }^{4,5}$ heart failure, ${ }^{4,6}$ hypertension, ${ }^{4,7}$ acute myocardial infarction, ${ }^{4,8}$ and arrhythmias. ${ }^{4,9,10}$ Various cohort studies have shown that OSA and OSA-associated symptoms may increase mortality ${ }^{11,12}$ and incidence of cardiovascular diseases by up to $50 \% .{ }^{12}$ Pathophysiological mechanisms involved in OSA are hypoxia due to collapsed upper airways and recurrent breathing against the closed or nearly closed oropharynx, which then results in negative intrathoracic pressure. A characterization of the haemodynamic changes during reduced intrathoracic pressure may, therefore, help to understand some of the cardiovascular consequences of OSA.

Other clinical applications of MM would be diseases associated with impaired relaxation of the myocardium, namely restrictive cardiomyopathy, constrictive pericarditis, or diastolic dysfunction. The expected changes in blood flow largely depend on intact physiological mechanisms involved in the extension of the myocardium and vessels through increase in venous return. If these mechanisms are damaged, real-time flow magnetic resonance imaging (MRI) would most likely show a decreased or even no reaction to the MM.

The purpose of this work was to evaluate the potential of a previously reported real-time phase-contrast flow MRI technique at high spatiotemporal resolution ${ }^{13,14}$ to dynamically monitor the immediate haemodynamic responses to the MM simultaneously in the ascending aorta (AA) and superior vena cava (SVC). Flow parameters for individual heartbeats, such as peak mean velocity and flow volume per heartbeat, were obtained continuously during free breathing, reduced intrathoracic pressure, and recovery.

\section{Materials and methods}

\section{Subjects}

Twenty healthy and, on average, young volunteers participated in the study (Table 1). Subjects had no history of respiratory or cardiovascular disease and were without contraindication for MRI. The study was approved by the institutional review board and written informed consent was obtained from each subject before MRI. The study was in consent with the Declaration of Helsinki.

\section{Real-time phase-contrast flow MRI}

As demonstrated in Fig 1 blood flow was measured in the $\mathrm{AA}$ and SVC at the same time using a single transverse plane perpendicular to the AA at the level of the right pulmonary artery. All studies were performed on a clinical $3 \mathrm{~T}$ MRI system (Tim Trio, Siemens Healthcare, Erlangen, Germany).

Flow evaluations in real time were accomplished with the use of a highly undersampled radial fast low-angle shot (FLASH) sequence with image reconstruction by regularized non-linear inversion (NLINV). ${ }^{15,16}$ The NLINV method was modified for phase-contrast flow MRI to yield phasesensitive reconstructions of two series of differently flowencoded images, while the subsequent calculation of velocity-encoded phase-contrast maps was performed without any temporal filter. ${ }^{13,14}$ Magnitude images and phase-contrast maps were obtained online using a server with $2 \times 4$ graphics processing units (sysGen/TYAN Octuple-GPU, 2x Intel Westmere E5620 processor, 48 GB RAM, Sysgen, Bremen, Germany) which was fully integrated into the reconstruction pipeline of the commercial MRI system. ${ }^{17,18}$

Two sequential images with and without a bipolar velocity-encoding gradient $(\mathrm{VENC}=200 \mathrm{~cm} / \mathrm{s}$ ) were

Table 1

Subjects.

\begin{tabular}{ll}
\hline Age, years & $33.9 \pm 12.8$ \\
Range & $22-59$ \\
Weight, kg & $71.5 \pm 13.7$ \\
Height, m & $1.76 \pm 0.11$ \\
Body mass index, kg/m & $22.8 \pm 2.6$ \\
Cardiovascular risk factors & $n=1^{\mathrm{a}}$ \\
Gender & 12 male, 8 female \\
\hline
\end{tabular}

a One subject with nicotine abuse. 


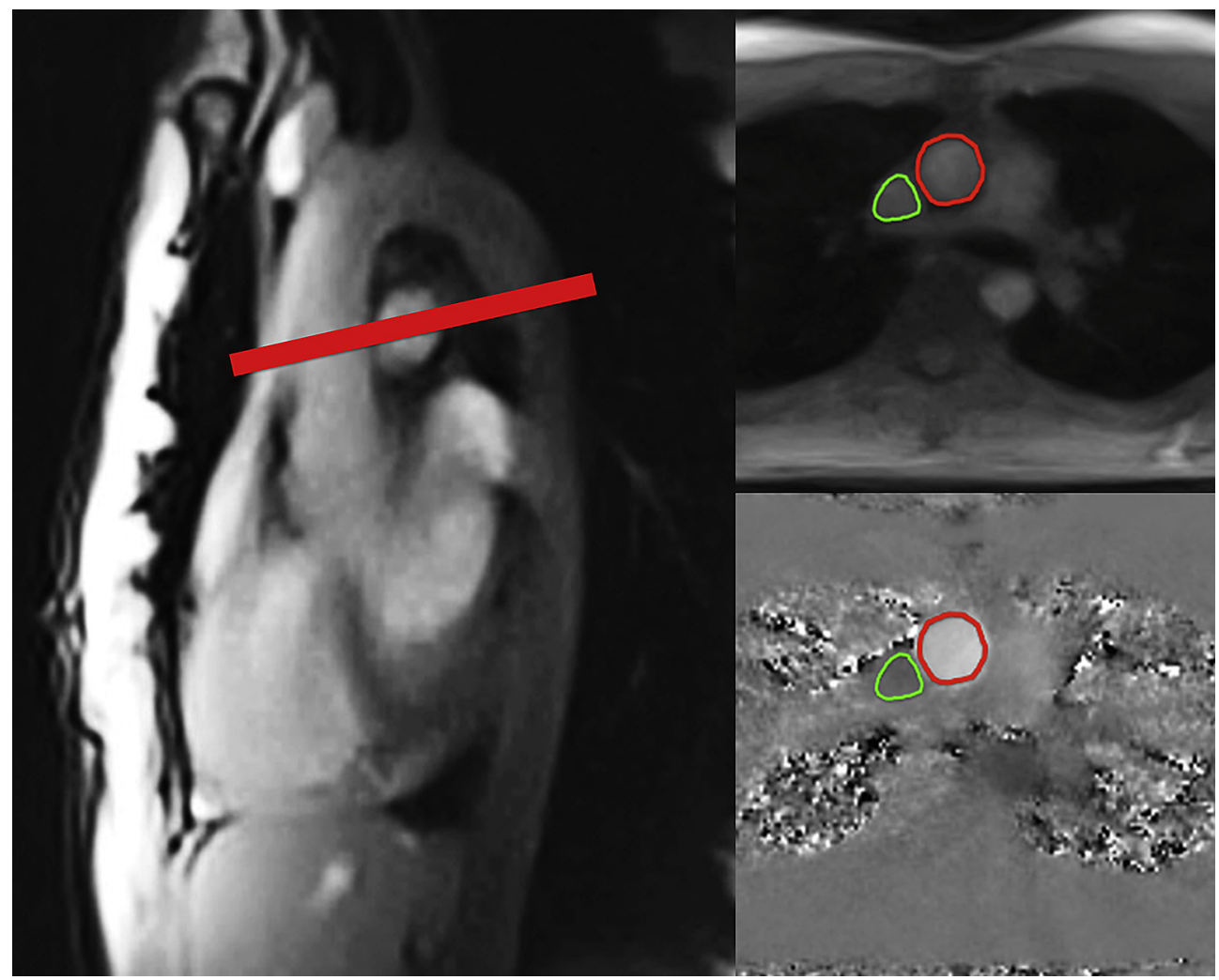

Figure 1 (Left) Section orientation and (right) segmented AA and SVC in a systolic real-time magnitude image (top) and phase-contrast flow map (bottom) in an angulated transverse view.

obtained from only seven spokes within an acquisition time of $20 \mathrm{~ms}$ each. ${ }^{14}$ Experimental parameters were: repetition time $(\mathrm{TR})=2.86 \mathrm{~ms}$, echo time $(\mathrm{TE})=1.93 \mathrm{~ms}, 10^{\circ}$ flip angle, $192 \times 192 \mathrm{~mm}^{2}$ field of view, and $144 \times 144$ acquisition matrix (no aliasing because of radial acquisitions and twofold oversampling), $1.33 \times 1.33 \mathrm{~mm}^{2}$ nominal in-plane resolution, and $6 \mathrm{~mm}$ section thickness. Although not required for data acquisition, the electrocardiography (ECG) was co-registered to facilitate the post-acquisition flow analysis using preliminary experimental software that allowed for treatment of multiple cardiac cycles (Qflow 5.4, MEDIS, Leiden, The Netherlands). Segmentations of the AA and SVC employed a semi-automatic finding of contours (compare Fig 1) with manual corrections if necessary.

\section{MM and pressure measurement}

In order to reduce the intrathoracic pressure, subjects were asked to inhale against a nearly closed epiglottis after low expiration. They had to maintain a negative pressure of at least $30 \mathrm{mmHg}$ for a period of $10 \mathrm{~s}$. Former studies defined this value as effective to induce haemodynamic changes in the cardiac outflow tract. ${ }^{19,20}$ Before the examination, each subject had to practice the MM outside the MRI machine. The pressure was measured by a digital manometer (GMSD 2 BR, Greisinger electronic GmbH, Regenstauf, Germany) at a sampling rate of $1 \mathrm{kHz}$. It was placed outside the magnet room and connected with a long flexible tube to a mouthpiece mounted on a tubus (Oxford
Tracheal Tube 7.0, closed system). Previous work confirmed a high positive correlation between intra-oral and intrapleural pressure levels during periods of increased or decreased intrathoracic pressure. ${ }^{21}$ The protocol for realtime flow MRI of the MM was repeated three times per subject and comprised normal breathing (10 s), reduced intrathoracic pressure (10 s), and normal breathing during recovery (20 s). During the examinations, subjects wore LCD glasses and received real-time visual feedback about the temporal progress of the protocol and the actually achieved pressure level. For later analysis and correlation of MM phases with serial phase-contrast flow maps, the elapsed time between the start of the manoeuvre and the recorded pressure curve, was used as real-time flow MRI acquisitions and pressure measurements were simultaneously started.

\section{Statistical analysis}

Functional evaluations of flow in the AA and SVC involved vessel areas, mean flow velocities spatially averaged over the vessel lumen (in particular, its peak value per heartbeat), flow volumes per heartbeat, and heart rate. Values are given as mean with standard deviation (SD) where appropriate. The analysis (AA and SVC) of each dataset (40 s duration $=1000$ images and phase-contrast maps, respectively) took approximately $30 \mathrm{~min}$. For statistical analyses of individual haemodynamic adjustments, the datasets were subdivided into five parts: baseline with normal breathing (10 heartbeats), early strain of reduced 
intrathoracic pressure (first three heartbeats during MM), late strain (last three heartbeats during MM), early recovery (first three heartbeats after the end of the MM), and late recovery (10 heartbeats during normal breathing).

Parameter distributions were tested by d'Agostino and Pearson's omnibus normality test. Repeated-measurement one-way analysis of variance (ANOVA) with Greenhouse-Geisser correction followed by Tukey's multiple comparisons test was performed using GraphPad Prism version 6.0d for Mac OS X (GraphPad Software, La Jolla, CA, USA) to compare haemodynamic parameters during normal breathing and MM, $p$-values $<0.05$ were considered statistically significant.

\section{Results}

Real-time flow MRI measurements during normal breathing and MM were technically successful in all cases. No cases of cardiovascular emergency, interventions, or sudden terminations of the examination occurred. However, eight of 20 subjects had to be excluded due to an incorrect pressure performance. In these cases, subjects did not achieve a constant pressure reduction of at least $30 \mathrm{mmHg}$, but instead presented with a highly variable pressure curve, which was often dominated by a very strong and rapid initial pressure decrease followed by a continuous return to almost normal levels. For example, one subject reached $-100 \mathrm{mmHg}$ during the first $2-3 \mathrm{~s}$, which then led to exhaustion and a subsequent increase to zero pressure. In addition, in a few cases $(\sim 5 \%)$ individual cardiac cycles were omitted, because the co-registered ECG was affected by gradient interference and failed to adequately set time stamps into the DICOM images, which in turn precluded the semi-automated analysis of flow parameters.

Quantitative haemodynamic adjustments in response to reduced intrathoracic pressure and recovery are summarized in Table 2. They are given in absolute values as well as percentage changes relative to the initial phase of normal breathing. Statistical comparisons of intrasubject changes relative to baseline were restricted to early strain and late recovery phases to ensure at least 23 successful serial events. An example for a single subject with good pressure performance is shown in Fig 2. Apart from changes that are caused by the MM, normal breathing periods exhibited pronounced respiration-induced modulations of velocity and flow, which lead to increased flow during inspiration due to lower intrathoracic pressure.

The pressure changes associated with the MM alter the peak mean velocity and flow per heartbeat in both the AA and SVC. When considering mean intrasubject differences averaged across subjects and manoeuvres (Table 2), and despite pronounced interindividual variability, the results for the initial phase of reduced intrathoracic pressure (early strain) revealed significant $-24 \%$ and $-25 \%$ decreases of the peak mean flow velocity and blood flow per heartbeat in the $\mathrm{AA}$, respectively. These observations were accompanied by a significant $+28 \%$ increase of peak mean velocity in the SVC during the same period and an even more pronounced $+49 \%$ increase of flow per heartbeat, which includes an increased SVC lumen during this phase. The induced flow alterations in the AA and SVC largely normalized during sustained MM (late strain in Table 2) except for a reverse $33 \%$ flow decrease in the SVC during late strain/early recovery. On average, the heart rate of the subjects was transiently elevated by $12 \%$ after the start (early strain) and $10 \%$ after the end (early recovery) of the MM.

\section{Discussion}

Real-time flow MRI at high spatiotemporal resolution quantitatively depicted rapid haemodynamic adjustments in the AA and SVC that are elicited by a reduced intrathoracic pressure and the subsequent return to normal breathing. The initial increase in SVC flow most likely reflects enhanced venous inflow into the right heart and atrium. Decreased AA flow might result from decreased pulmonary venous return and subsequent decreased preload during the initial phase. The slight increases of AA flow during late strain and, more pronounced, during recovery possibly reflect a compensatory increase in pulmonary flow, after the increase in systemic venous return passed through the pulmonary circulation. These results are in general agreement with previous studies of the MM using various techniques including echocardiography, arterial pulse-wave

Table 2

Real-time flow MRI in the ascending aorta and superior vena cava before, during and after the Mueller manoeuvre.

\begin{tabular}{|c|c|c|c|c|c|}
\hline & Normal breathing $n=23$ & Early strain $n=24$ & Late strain $n=18$ & Early recovery $n=17$ & Late recovery $n=24$ \\
\hline \multicolumn{6}{|l|}{ Ascending aorta } \\
\hline Peak mean velocity, $\mathrm{cm} / \mathrm{s}$ & $55 \pm 9$ & $41 \pm 6$ & $57 \pm 9$ & $60 \pm 12$ & $60 \pm 12$ \\
\hline Intrasubject differences & $100 \%$ & $76 \pm 9 \% * *$ & $103 \pm 9 \%$ & $107 \pm 11 \%$ & $110 \pm 10 \% *$ \\
\hline Flow, ml/beat & $86 \pm 12$ & $64 \pm 11$ & $91 \pm 14$ & $92 \pm 15$ & $91 \pm 15$ \\
\hline Intrasubject differences & $100 \%$ & $75 \pm 9 \% * *$ & $103 \pm 7 \%$ & $104 \pm 11 \%$ & $105 \pm 6 \% *$ \\
\hline \multicolumn{6}{|l|}{ Superior vena cava } \\
\hline Peak mean velocity, cm/s & $32 \pm 6$ & $40 \pm 7$ & $30 \pm 9$ & $32 \pm 9$ & $33 \pm 7$ \\
\hline Intrasubject differences & $100 \%$ & $128 \pm 25 \% * *$ & $92 \pm 19 \%$ & $99 \pm 18 \%$ & $104 \pm 13 \%$ \\
\hline Flow, ml/beat & $26 \pm 7$ & $38 \pm 11$ & $19 \pm 11$ & $29 \pm 8$ & $27 \pm 8$ \\
\hline Intrasubject differences & $100 \%$ & $149 \pm 44 \% * *$ & $67 \pm 26 \%$ & $105 \pm 29 \%$ & $103 \pm 14 \%$ \\
\hline Heart rate, beats/min & $73 \pm 7$ & $82 \pm 11$ & $76 \pm 10$ & $79 \pm 11$ & $71 \pm 7$ \\
\hline Intrasubject differences & $100 \%$ & $112 \pm 9 \% * *$ & $105 \pm 7 \%$ & $110 \pm 9 \%$ & $98 \pm 8 \%$ \\
\hline
\end{tabular}

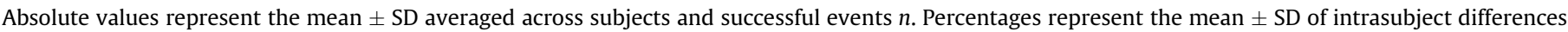
relative to normal breathing. Statistical analyses only refer to "Early strain" and "Late recovery" versus "Normal breathing": ${ }^{* *} p<0.0001$, ${ }^{*} p<0.01$. 


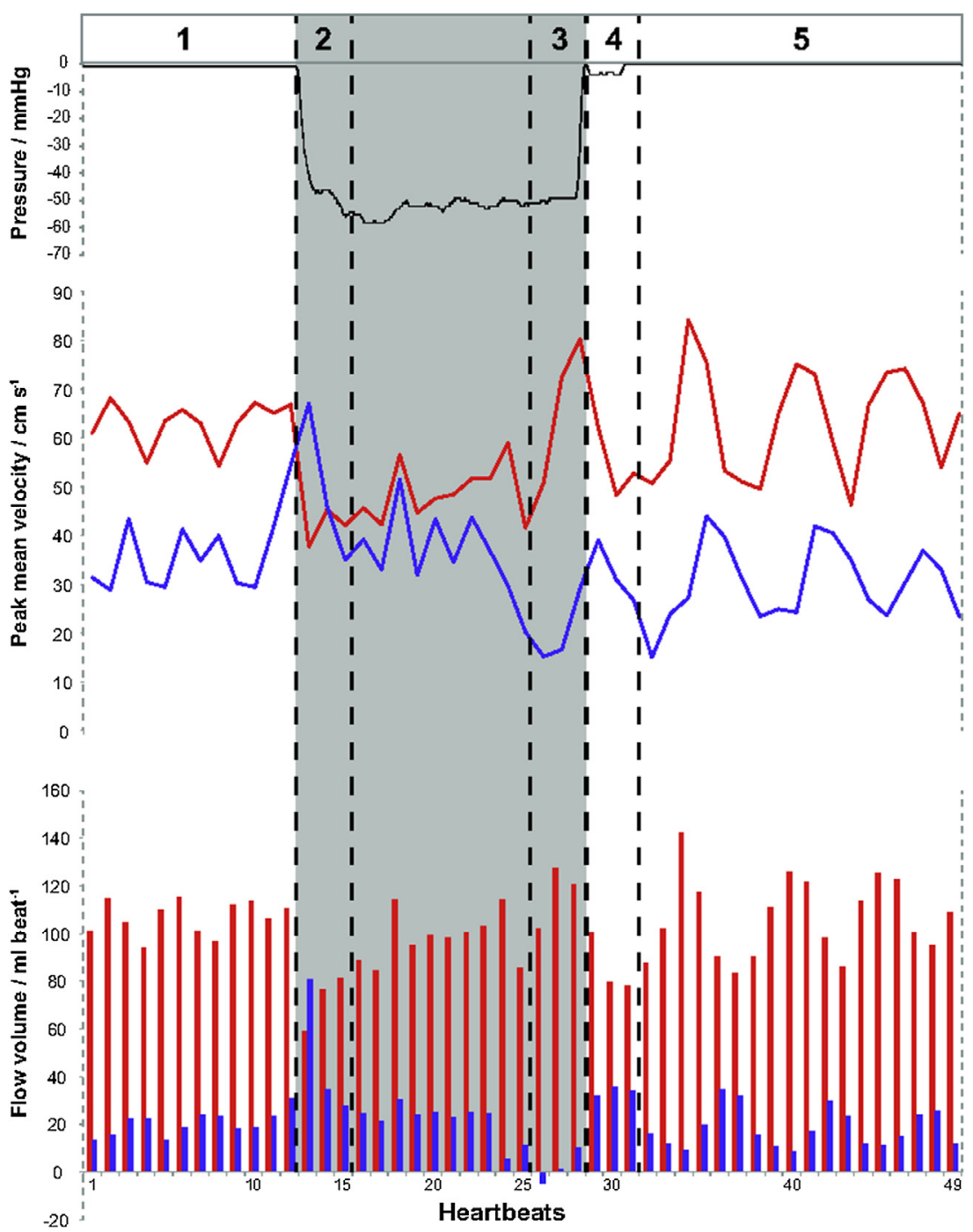

Figure 2 Intrathoracic pressure, peak mean flow velocity, and flow per heartbeat in the AA (red) and SVC (blue) of a 25-year-old healthy volunteer before, during, and after MM (grey area). Phase $1=$ normal breathing, $2=$ early and $3=$ late strain $(\mathrm{MM}), 4=$ early and $5=$ late recovery (normal breathing).

analysis, and electrical impedance cardiography., ${ }^{1,419,22-25}$ For example, negative intrathoracic pressure was associated with a decrease of cardiac stroke volume ${ }^{1,20,24,25}$ by $-29 \%$ to $-11 \%$ for different protocols and groups of subjects. Thus, previous and present results demonstrate a reduced left-ventricular systolic performance during reduced intrathoracic pressure.

Technically, real-time flow MRI overcomes a number of limitations that are specific to echocardiography. First of all, echocardiography does not allow for the measurement of two or more vessels at the same time. Moreover, the procedure requires a good and stable acoustic window, which is often impossible to achieve in overweight adults or during forced breathing or breath-hold protocols; the latter problem may even preclude continuous flow recordings throughout a respective manoeuvre. Finally, the accuracy of flow assessments by echocardiography relies on velocity measurements in only a small portion of the vessel and assumptions about the shape and dimension of its lumen.
These parameters usually vary during the cardiac cycle as well as in response to induced pressure changes. In contrast, MRI-based flow determinations measure and integrate the full two-dimensional velocity distribution over the true vessel lumen at every single point in time (here every $40 \mathrm{~ms}$ ).

The most challenging part of the proposed method and a potential limitation for clinical use is a reliable conduction of the MM itself. Subjects tended to initially overshoot the negative target pressure of $-30 \mathrm{mmHg}$, which led to an even stronger increase of flow and peak mean velocities. In fact, more than $50 \%$ of all manoeuvres showed this distinct overshoot, which led to exclusion from the further analysis. It remains to be seen whether a more careful pre-scan training and better feedback via LCD glasses during MRI will help to overcome these problems in order to maintain clinical applicability. It should also be noted that the present study was performed at a magnetic field strength of $3 \mathrm{~T}$, which offers adequate signal-to-noise for real-time phase- 
contrast flow MRI. It remains to be seen whether modern 1.5 T MRI systems with optimized gradients and multielement receiver coils for body measurements will reach the required image quality.

In summary, real-time phase-contrast flow MRI successfully unravelled rapid haemodynamic adjustments in response to the $\mathrm{MM}$, quantitatively and simultaneously in two great thoracic vessels. In future, abnormal flow responses to this manoeuvre might be helpful in studies of patients with OSA, offering new insights into the pathogenesis of OSA-associated cardiovascular diseases. In a more general clinical context, immediate access to the physiological consequences of intrathoracic pressure changes by real-time flow MRI emerges as a new tool for the simultaneous measurement of interdependent arterial and venous flow in the right and left heart.

\section{Acknowledgement}

We thank the DFG (project LO 1773/1) for financial support. S.Z. and J.F. hold a patent regarding the real-time MRI acquisition and reconstruction technique used here.

\section{References}

1. Condos Jr WR, Latham RD, Hoadley SD, et al. Hemodynamics of the Mueller maneuver in man: right and left heart micromanometry and Doppler echocardiography. Circulation 1987;76:1020-8.

2. Rothman A, Goldberger AL. Aids to cardiac auscultation. Ann Intern Med 1983;99:346-53.

3. Marin JM, Carrizo SJ, Vicente E, et al. Long-term cardiovascular outcomes in men with obstructive sleep apnoea-hypopnoea with or without treatment with continuous positive airway pressure: an observational study. Lancet 2005;365(9464):1046-53.

4. Koshino Y, Villarraga HR, Orban M, et al. Changes in left and right ventricular mechanics during the Mueller maneuver in healthy adults: a possible mechanism for abnormal cardiac function in patients with obstructive sleep apnea. Circ Cardiovasc Imaging 2010;3:282-9.

5. Gami AS, Howard DE, Olson EJ, et al. Day-night pattern of sudden death in obstructive sleep apnea. N Engl J Med 2005;352:1206-14.

6. Romero-Corral A, Somers VK, Pellikka PA, et al. Decreased right and left ventricular myocardial performance in obstructive sleep apnea. Chest 2007; 132:1863-70.

7. Peppard PE, Young T, Palta M, et al. Prospective study of the association between sleep-disordered breathing and hypertension. $N$ Engl J Med 2000;342:1378-84.

8. Kuniyoshi FH, Garcia-Touchard A, Gami AS, et al. Day-night variation of acute myocardial infarction in obstructive sleep apnea. J Am Coll Cardiol 2008;52:343-6.
9. Mehra R, Benjamin EJ, Shahar E, et al. Association of nocturnal arrhythmias with sleep-disordered breathing: The Sleep Heart Health Study. Am J Resp Crit Care Med 2006;173:910-6.

10. Koshino Y, Satoh M, Katayose Y, et al. Association of sleep-disordered breathing and ventricular arrhythmias in patients without heart failure. Am J Cardiol 2008;101:882-6.

11. Marshall NS, Wong KK, Cullen SR, et al. Sleep apnea and 20-year followup for all-cause mortality, stroke, and cancer incidence and mortality in the Busselton health study cohort. J Clin Sleep Med 2014;10:355-62.

12. Kendzerska T, Gershon AS, Hawker G, et al. Obstructive sleep apnea and risk of cardiovascular events and all-cause mortality: a decade-long historical cohort study. PLoS Med 2014;11:e1001599.

13. Joseph AA, Merboldt KD, Voit D, et al. Real-time phase-contrast MRI of cardiovascular blood flow using undersampled radial fast low-angle shot and nonlinear inverse reconstruction. NMR Biomed 2012;25:917-24.

14. Joseph A, Kowallick JT, Merboldt KD, et al. Real-time flow MRI of the aorta at a resolution of 40 msec. J Magn Reson Imaging 2014;40:206-13.

15. Uecker M, Zhang S, Voit D, et al. Real-time MRI at a resolution of $20 \mathrm{~ms}$. NMR Biomed 2010;23:986-94.

16. Uecker M, Zhang S, Voit D, et al. Real-time MRI: recent advances using radial FLASH. Imaging Med 2012;4:461-76.

17. Schaetz S, Uecker M. A multi-GPU programming library for real-time applications. In: Xiang Y, Stojmenovic I, Apduhan B, et al., editors. Algorithms and architectures for parallel processing. Berlin: Springer; 2012. pp. 114-28.

18. Frahm J, Schätz S, Untenberger $M$, et al. On the temporal fidelity of nonlinear inverse reconstructions for real-time MRI - the motion challenge. Open Med Imaging J 2014:1-7.

19. Robertson D, Stevens RM, Friesinger GC, et al. The effect of the Valsalva maneuver on echocardiographic dimensions in man. Circulation 1977;55:596-662.

20. Bradley TD, Hall MJ, Ando S, et al. Hemodynamic effects of simulated obstructive apneas in humans with and without heart failure. Chest 2001; 119:1827-35.

21. Elisberg EI, Goldberg H, Snider GL. Value of intraoral pressure as a measure of intrapleural pressure. J Appl Physiol 1951;4:171-6.

22. Paulev PE, Honda Y, Sakakibara Y, et al. Brady- and tachycardia in light of the Valsalva and the Mueller maneuver (apnea). Japan J Physiol 1988;38:507-17.

23. Carrasco-Sosa S, Guillen-Mandujano A. Response patterns of arterial pressure and heart period to Mueller manoeuvre and their comparison to those of Valsalva manoeuvre. Clin Physiol Funct Imaging 2012;32:253-61.

24. Orban M, Bruce CJ, Pressman GS, et al. Dynamic changes of left ventricular performance and left atrial volume induced by the Mueller maneuver in healthy young adults and implications for obstructive sleep apnea, atrial fibrillation, and heart failure. Am J Cardiol 2008;102:1557-61.

25. Viscor I, Jurak P, Vondra V, et al. Stroke volume during mueller maneuver measured by impedance cardiography in patients with mitral regurgitation. Comp Cardiol 2009;2009:749-51. 